\title{
CT angiography at 24 months demonstrates durability of EVAR with the use of chimney grafts for pararenal aortic pathologies
}

\author{
Donas, Konstantinos P ; Pecoraro, Felice ; Bisdas, Theodosios ; Lachat, Mario ; Torsello, Giovanni ; \\ Rancic, Zoran ; Austermann, Martin ; Mayer, Dieter ; Pfammatter, Thomas ; Puchner, Stefan
}

\begin{abstract}
PURPOSE: To present the 24-month radiological follow-up data for patients with pararenal aortic pathologies treated with chimney and periscope grafts during endovascular repair. METHODS: Between January 2008 and December 2011, 124 high-risk patients with complex pararenal aortic pathologies were treated using the chimney technique at 2 European vascular and cardiovascular centers with advanced experience of the described technique. In particular, 50 patients were treated at Site 1 and 74 at Site 2. Forty (32.2\%) patients (32 men; mean age $79.2 \pm 4.9$ years) completed computed tomographic angiography follow-up at 24 months postoperatively. RESULTS: The overall technical success was $100 \%$, and the early- and midterm procedure-related mortality was $0 \%$. Three $(2.4 \%)$ patients had a perioperative type Ia endoleak that persisted; two were treated by transbrachial perigraft embolization and cuff implantation. The last patient is under radiological surveillance due to a "low-flow" type Ia endoleak and stable sac size. A type II endoleak was detected in 7 (5.6\%) patients. During the 2-year follow-up, significant shrinkage $(>5 \mathrm{~mm} ; \mathrm{n}=22)$ or stable aneurysm diameter $(\mathrm{n}=14)$ was seen in $36(90 \%)$ of the cases. Overall, mean aneurysm sac shrinkage was $12 \%(\mathrm{p}=0.002)$ and $10 \%(\mathrm{p}=0.014)$ for the 2 centers, respectively (overall $\mathrm{p}=0.008$ ). The causes for sac progression in the $4(10 \%)$ patients were a type Ia endoleak, 2 type II endoleaks, and endotension. CONCLUSION: The present study demonstrates that the use of chimney and/or periscope endografts for pararenal aortic pathologies achieves and maintains successful exclusion of the aneurysm in $90 \%$ of the cases at 24 months of radiological follow-up. In centers experienced with this approach, the chimney technique may represent a reliable therapeutic modality in selected patients.
\end{abstract}

DOI: https://doi.org/10.1583/12-4029.1

Posted at the Zurich Open Repository and Archive, University of Zurich

ZORA URL: https://doi.org/10.5167/uzh-85125

Journal Article

Published Version

Originally published at:

Donas, Konstantinos P; Pecoraro, Felice; Bisdas, Theodosios; Lachat, Mario; Torsello, Giovanni; Rancic, Zoran; Austermann, Martin; Mayer, Dieter; Pfammatter, Thomas; Puchner, Stefan (2013). CT angiography at 24 months demonstrates durability of EVAR with the use of chimney grafts for pararenal aortic pathologies. Journal of Endovascular Therapy, 20(1):1-6.

DOI: https://doi.org/10.1583/12-4029.1 


\title{
CT Angiography at 24 Months Demonstrates Durability of EVAR With the Use of Chimney Grafts for Pararenal Aortic Pathologies
}

\author{
Konstantinos P. Donas, MD, PhD ${ }^{1}$; Felice Pecoraro, MD²; Theodosios Bisdas, MD; \\ Mario Lachat, $\mathrm{MD}^{2}$; Giovanni Torsello, MD, $\mathrm{PhD}^{1}$; Zoran Rancic, MD, $\mathrm{PhD}^{2}$; \\ Martin Austermann, MD, PhD ${ }^{1}$; Dieter Mayer, MD²; Thomas Pfammatter, MD; \\ and Stefan Puchner, MD ${ }^{4}$
}

${ }^{1}$ Clinic for Vascular and Endovascular Surgery, Münster University Hospital and St. Franziskus Hospital, Münster, Germany. ${ }^{2}$ Clinic for Cardiovascular Surgery and ${ }^{3}$ Institute of Diagnostic Radiology, Zürich University Hospital, Zürich, Switzerland. ${ }^{4}$ Department of Radiology, Division of Cardiovascular and Interventional Radiology, Medical University Vienna, Austria.

Purpose: To present the 24-month radiological follow-up data for patients with pararenal aortic pathologies treated with chimney and periscope grafts during endovascular repair. Methods: Between January 2008 and December 2011, 124 high-risk patients with complex pararenal aortic pathologies were treated using the chimney technique at 2 European vascular and cardiovascular centers with advanced experience of the described technique. In particular, 50 patients were treated at Site 1 and 74 at Site 2. Forty (32.2\%) patients (32 men; mean age $79.2 \pm 4.9$ years) completed computed tomographic angiography follow-up at 24 months postoperatively.

Results: The overall technical success was $100 \%$, and the early- and midterm procedurerelated mortality was $0 \%$. Three $(2.4 \%)$ patients had a perioperative type la endoleak that persisted; two were treated by transbrachial perigraft embolization and cuff implantation. The last patient is under radiological surveillance due to a "low-flow" type la endoleak and stable sac size. A type II endoleak was detected in $7(5.6 \%)$ patients. During the 2-year follow-up, significant shrinkage ( $>5 \mathrm{~mm} ; \mathrm{n}=22$ ) or stable aneurysm diameter $(\mathrm{n}=14)$ was seen in $36(90 \%)$ of the cases. Overall, mean aneurysm sac shrinkage was $12 \%(p=0.002)$ and $10 \%(p=0.014)$ for the 2 centers, respectively (overall $p=0.008)$. The causes for sac progression in the $4(10 \%)$ patients were a type la endoleak, 2 type II endoleaks, and endotension.

Conclusion: The present study demonstrates that the use of chimney and/or periscope endografts for pararenal aortic pathologies achieves and maintains successful exclusion of the aneurysm in $90 \%$ of the cases at 24 months of radiological follow-up. In centers experienced with this approach, the chimney technique may represent a reliable therapeutic modality in selected patients.

J Endovasc Ther. 2013;20:1-6

Key words: endovascular aneurysm repair, chimney graft, periscope graft, pararenal aortic pathologies, aortic aneurysm, para-anastomotic aneurysm, ruptured aneurysm, stent-graft, balloon-expandable stent-graft, computed tomographic angiography, endoleak, renal arteries, superior mesenteric artery, sac shrinkage, sac expansion

The authors declare no association with any individual, company, or organization having a vested interest in the subject matter/products mentioned in this article.

Corresponding author: Konstantinos P. Donas, MD, PhD, Asst. Prof. Vascular Surgery, Clinic for Vascular and Endovascular Surgery, St. Franziskus Hospital and University Hospital Münster, Hohenzollernring 72, D-48145 Münster, Germany. E-mail: k.donas@gmx.at 
Endovascular methods may offer alternative less invasive procedures for patients with severe comorbidities deemed unfit or at high risk for open repair. ${ }^{1}$ Several endovascular techniques have been proposed to ensure secure proximal fixation and to increase the applicability of endovascular repair for patients with pararenal aortic pathologies. One such technique is the chimney graft, in which a covered or bare metal stent is deployed parallel and outside of the aortic endograft,

See commentary page 7

inflow upward (i.e., periscopes) or downward (i.e., chimneys), to retain or rescue blood flow into aortic branches along the sealing zones. ${ }^{2,3}$ The published experience with this minimally invasive aortic branch preservation technique does not allow drawing robust conclusions, $^{2,3}$ primarily because mid- and long-term results of this novel technique are lacking. ${ }^{2}$

The current article aims to present midterm (24-month) results of contrast-enhanced computed tomographic angiography (CTA) in a large cohort of patients with pararenal aortic pathologies treated by means of chimney and periscope endografts.

\section{METHODS}

Between January 2008 and December 2011, 124 high-risk patients with pararenal aortic pathologies were treated using the endovascular chimney technique in conjunction with endovascular aneurysm repair (EVAR) at 2 high-volume European centers. In particular, 50 patients were treated at Site 1 (Münster, Germany) and 74 patients at Site 2 (Zürich, Switzerland). Forty $(32.2 \%)$ of these patients (32 men; mean age $79.2 \pm 4.9$ years) completed radiological follow-up at 24 months $(n=14$ at site 1 and 26 at site 2; Table 1). Patients treated with chimneys or periscopes for arch and/or thoracoabdominal aneurysms were excluded from this analysis (27 at Site 1 and 4 at Site 2).

\section{Chimney Technique}

Generally, chimney grafts were implanted through a transbrachial or transaxillary ap-

\begin{tabular}{lr}
\hline \multicolumn{2}{c}{ TABLE } \\
Patient Characteristics and Comorbidities \\
\hline Age, y & $79.2 \pm 4.9$ \\
Men & $32(80.0 \%)$ \\
Hypertension & $23(57.5 \%)$ \\
Diabetes mellitus & $12(30.0 \%)$ \\
Chronic obstructive pulmonary & $11(27.5 \%)$ \\
$\quad$ disease & \\
Myocardial infarction / coronary & $9(22.5 \%)$ \\
$\quad$ artery disease & \\
Renal insufficiency & $10(25.0 \%)$ \\
Hypercholesterolemia & $6(15.0 \%)$ \\
Degenerative aneurysm & $29(72.5 \%)$ \\
Endoleak type la & $7(17.5 \%)$ \\
Para-anastomotic aneurysm & $4(10.0 \%)$ \\
Neck length 0-3 mm & $32(80.0 \%)$ \\
Neck length 4-9 mm & $8(20.0 \%)$ \\
Symptomatic & $13(32.5 \%)$ \\
Ruptured aneurysm & $4(10.0 \%)$ \\
\hline
\end{tabular}

Continuous data are presented as the means \pm standard deviation; categorical data are given as the counts (percentage).

proach and periscope grafts via a transfemoral access. The selection of the access vessel was based on several factors, such as endoluminal anatomy and disease of the subclavian/axillary and humeral artery and of the aortic arch. At Site 2, occasionally and in the presence of extensive atherosclerotic disease of the arch, chimney grafts were introduced and deployed transfemorally like a periscope graft and pushed up to end as a chimney graft.

\section{EVAR With Chimney Technique}

All procedures at Site 1 were performed in a hybrid operating room under fluoroscopic control (Axiom Artis FA; Siemens AG, Forchheim, Germany) with a totally percutaneous transfemoral approach using the 10-F Prostar $\mathrm{XL}$ vascular closure device (Abbott Vascular, Abbott Park, IL, USA). The chimney grafts were advanced in the renovisceral aortic segment via an open or percutaneous brachial approach and use of a 6-F or 7-F Shuttle sheath (Cook Inc., Bloomington, IN, USA); a Rosen wire was preferred for catheterization of the renovisceral vessels due to its atraumatic $\mathrm{J}$ tip. The chimney stent(s) (balloonexpandable Advanta stent-grafts; Atrium 
Medical, Hudson, NH, USA) were deployed after the main body stent-graft to preserve flow to over-stented renal or superior mesenteric arteries.

At Site 2, the chimney technique was performed in similar fashion in a hybrid operating room under fluoroscopic control (Philips Medical Systems, Inc., Shelton, CT, USA) or an angiographic suite (Artiszeego; Siemens AG). Access to the aorta was obtained with a femoral cutdown and either a surgiclose technique ${ }^{5}$ or by using one or several ProGlide Suture-Mediated Closure devices (Abbott Vascular, Redwood City, CA, USA). The renovisceral arteries were accessed through the axillary and femoral routes with a 45-cm Arrow sheath (Arrow International Inc., Reading, PA, USA), a 5-F Chuang visceral reverse curve catheter (Cook Inc.), and a Rosen wire. Chimney and periscope grafts were mostly deployed into the renovisceral arteries before aortic stent-graft deployment. After balloon molding of the chimney and periscope grafts, the balloon was reinflated inside the chimney and/or periscope graft, preferably inside the renovisceral branch, and gentle pullback traction was applied to the balloon catheter to achieve a parallel graft design before the aortic stentgraft was deployed. The preferred stent-graft for chimney or periscope grafts was the Viabahn (W.L. Gore \& Associates, Flagstaff, AZ, USA) at Site 2.

Sites 1 and 2 restricted the use of chimney grafts to pararenal aortic pathologies involving 1 or 2 aortic side branches, whereas for more demanding anatomies with involvement of $>2$ side branches, Site 1 preferred the use of fenestrated endografts and Site 2 hybrid repair.

\section{Definitions}

Patients were classified as high risk for surgical repair if they had an American Society of Anesthesiologists score $\geq 3$ or suffered from $>3$ serious cardiovascular comorbidities: chronic obstructive lung disease, congestive heart failure, coronary artery occlusive disease, previous myocardial infarction, history of coronary artery stent or bypass, or prior aortic and/or aortoiliac pro- cedures. ${ }^{2}$ All symptomatic aortic pathologies were considered at increased risk.

Pararenal aortic pathologies included degenerative aortic aneurysms $(n=29)$, type la endoleaks $(n=7)$, and para-anastomotic aneurysms $(n=4)$ with a very short $(<3-\mathrm{mm})$ or absent infrarenal neck extending to or involving at least 1 renal artery (i.e., in open repair a suprarenal or transrenal clamp would be necessary). The inadequate landing zone for the stent-graft below the renal vessels precluded conventional endovascular repair.

Technical success was achieved when the procedure was completed as intended, with devices deployed without any complication, target vessels were patent without flow limitation to the branches, and no high-flow type I or III endoleak. High-flow endoleak was present if contrast flowed immediately through the leak and at the same speed in the aneurysm sac and through the stent-graft. A low-flow endoleak was represented by contrast appearing in the aneurysm sac after a few seconds, generally after contrast had passed through the aortic stent-graft. Changes in aneurysm sac diameters were based on a 5-mm threshold. Renal function before and after the procedure was based on the glomerular filtration rate (GFR).

\section{Outcome Measures and Imaging}

Main outcomes of this study were (1) aneurysm sac size changes, (2) patency of the chimney grafts, and (3) endoleaks. Five types of endoleaks were defined based on the location (attachment of the aortic stent-graft, origin from aortic side branches including distal chimney/periscope landing zones), chimney graft-related due to disconnection/ migration of the stent-grafts, use of bare metal stents instead of covered stents, and finally on absence of contrast extravasation, also known as endotension.

All patients underwent CTA of the thorax, abdomen, and pelvis to delineate visceral and renal morphology and aortic and iliofemoral anatomy preoperatively, within the first 7 days postoperatively, at 3 months, and then annually. The CTA follow-ip data were culled from the radiologists' reports; these first readers had not been involved in the endo- 
A

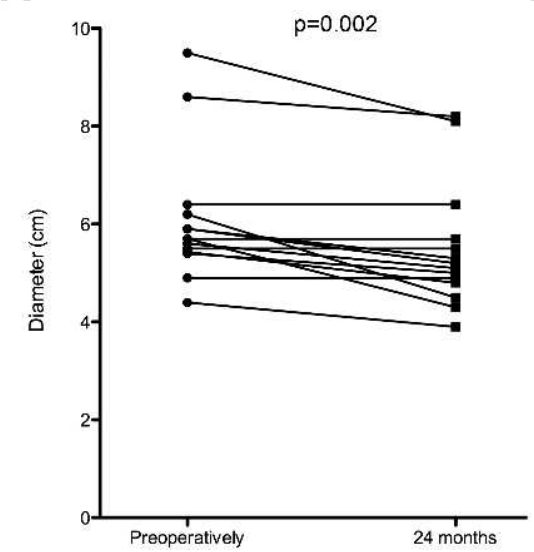

B

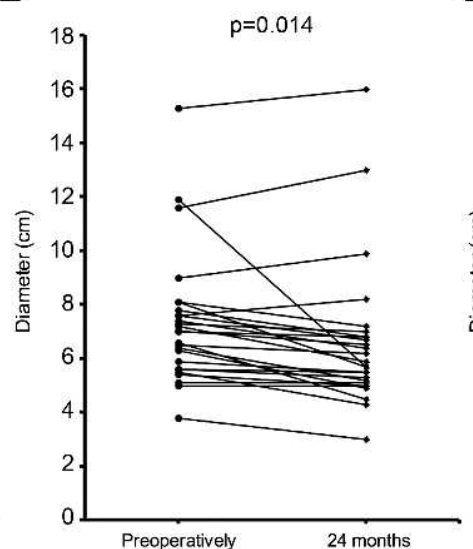

C

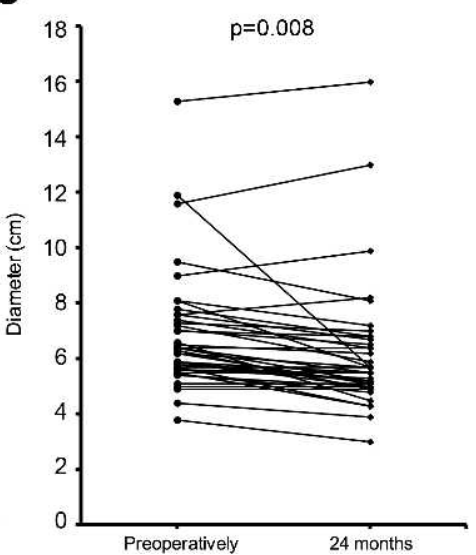

Figure 1 The variations between the preoperative and 24-month aneurysm diameters at (A) Site 1 (Münster, Germany), at (B) Site 2 (Zürich, Switzerland), and (C) a comparison of all values.

vascular procedures. Re-reading of all CTAs of the cases treated at Site 1 was performed by an independent radiologist (S.P.) at the Vienna University Hospital. At Site 2, second consensus CTA reading of their cases was performed by 2 vascular surgeons (M.L., F.P.) and a radiologist (T.P.) with extensive experience in endovascular aortic surgery and CTA interpretation.

\section{Statistical Analysis}

Continuous data are presented as the means \pm standard deviation; categorical data are given as the counts (percentage). The paired $t$ test was used to compare the aneurysm diameter prior to and after chimney graft implantation; $\mathrm{p}<0.05$ was considered statistically significant. All analyses were performed using GraphPad Prism (version 5.0; GraphPad Software Inc., San Diego, CA, USA).

\section{RESULTS}

Primary technical success was achieved in 40 $(100 \%)$ cases. The overall procedure-related mortality was zero. Mean length and mean diameter of the 73 parallel grafts deployed were $4.4 \pm 2.2 \mathrm{~cm}$ and $6.2 \pm 1.0 \mathrm{~mm}$ at Site 1, respectively, and $7.2 \pm 4.6 \mathrm{~cm}$ and $6.9 \pm 1.0 \mathrm{~mm}$ at Site 2, respectively. Overall, a mean of 1.85 vessels per patient were revascularized; at
Site 1 , the mean was 1.1 vessels per patient (15 vessels/14 patients) while at Site 2, the mean was 2.2 vessels per patient (58 vessels/ 26 patients). The mean contrast volume was $130 \pm 33 \mathrm{~mL}$, and the mean time of radiation was $30.2 \pm 9.9$ minutes at Site 1 and $241 \pm 22$ $\mathrm{mL}$ and $55 \pm 2.2$, respectively, at Site 2 . At Site 1 , the mean GFR was $52.2 \pm 2.3 \mathrm{~mL} / \mathrm{min} / 1.73 \mathrm{~m}^{2}$ preoperatively and $55.2 \pm 3 \mathrm{~mL} / \mathrm{min} / 1.73 \mathrm{~m}^{2}$ postoperatively; at Site 2 , the mean values were $54.0 \pm 9$ preoperatively and $56.6 \pm 9$ postoperatively, respectively.

Three $(2.4 \%)$ patients with a primary perioperative endoleak showed persistent type la endoleak; 2 were treated by transbrachial perigraft embolization and cuff implantation. The last patient is under radiological surveillance due to a "low-flow" type la endoleak with no increase in the aneurysm sac size. A type II endoleak was detected in 7 (5.6\%) patients, originating from patent lumbar arteries in all cases.

Fourteen $(35 \%)$ of the 40 patients had a stable $( \pm 5 \mathrm{~mm})$ aneurysm diameter after 2 years $(6 / 14$ patients of Site 1 and $8 / 26$ of Site $2)$, while shrinkage of the aneurysm sac $(>5$ $\mathrm{mm}$ ) was observed in 8 of 14 at Site 1 and 14 of 26 at Site 2. Mean aneurysm sac shrinkage was $12 \%(p=0.002)$ for Site 1 and $10 \%$ $(p=0.014)$ for Site 2 ( $p=0.008$ for both sites combined, Fig. 1). While no aneurysm sac progression was observed at Site 1, 4 patients at Site 2 showed enlarging aneurysm diame- 


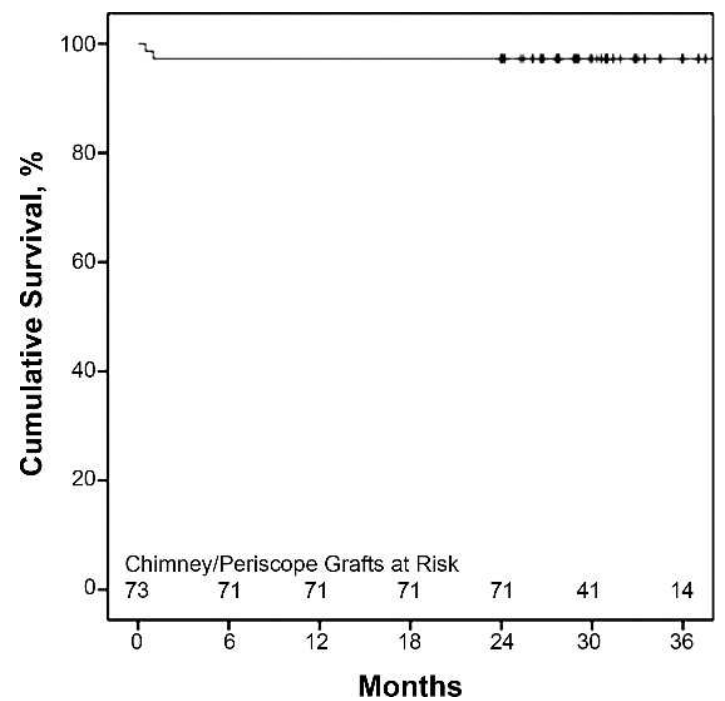

Figure 2 Cumulative primary patency of the chimney/periscope grafts.

ters (preoperative diameters of 90, 153, 116, and $76 \mathrm{~mm}$ ) of about $5 \mathrm{~mm}$. No endoleak could be detected in 1 of these patients. Another patient had a type la endoleak requiring a redo procedure, which consisted of transbrachial endoleak embolization and cuff implantation 19 months postoperatively. After this procedure, the aneurysm diameter remained stable, and no persistent type la endoleak was observed. The other 2 patients had type II endoleaks that were still under radiological surveillance.

Two $(2.7 \%)$ of the 73 parallel grafts were occluded (Fig. 2). In one case, the occluded chimney graft was explanted surgically 45 days postoperatively and an 8-mm iliorenal Dacron bypass graft was implanted. ${ }^{2}$ In the other case, the periscope graft occlusion was successfully managed by local thrombolysis and stenting 15 days postoperatively. One patient underwent repeated balloon angioplasty due to $>80 \%$ stenosis of a chimney graft.

\section{DISCUSSION}

The use of chimney or periscope endografts for complex abdominal pathologies is becoming more popular, ${ }^{1,6}$ but the absence of midterm results for this novel technique remains an issue. To inform the debate, the present article reports 24-month radiological follow-up in 40 patients from 2 centers experienced with the chimney and/or periscope EVAR technique. The use of such parallel grafts in EVAR led to maintained renal perfusion and significant midterm shrinkage of the aneurysm sac in both institutions, reflecting a valid therapeutic modality for patients with pararenal aortic pathologies who are unfit for open reconstruction or unsuitable for fenestrated endografting.

The balloon- or self-expanding covered stents employed in the chimney technique work well with today's flexible, low-profile abdominal aortic stent-grafts used to treat patients with tortuous iliac arteries or severely angulated necks. ${ }^{1}$ Another advantage of this technique is that the treatment is feasible in emergent cases involving the renovisceral vessels in which off-the-shelf devices have to be used. ${ }^{7,8}$ Size might be a limitation, as was experienced at Site 2.

A debatable topic remains the so-called "gutters" around the chimney grafts, which reflect the degree of conformation of the endograft around the chimney stent. The potential risk of type I endoleak, as well as the insufficient exclusion of the aneurysm sac and its growth, are justified concerns. However, the occurrence of persistent high-flow type I endoleaks ${ }^{2}$ in our study was rare and encountered only in Site 2 patients, perhaps because involvement of $>1$ side branch was more common at Site 2. At Site 1, the vascular surgeons preferred fenestrated devices in cases involving $>2$ side branches (e.g., in suprarenal pathologies). Nevertheless, Site 2's experience showed that there was no need for surgical conversion in cases of type la endoleak; the latter endoleaks could be successfully sealed by using coil or glue embolization.

The type of chimney/periscope graft (balloon-expandable or self-expanding device) seems not to be crucial for pararenal pathologies, especially those involving one renal artery. We recorded only 2 occlusions of the chimney grafts, one of each type. However, greater numbers of patients are needed to identify any possible difference. ${ }^{9}$ Use of covered devices can avoid endoleaks caused 
by porosity of the chimney stent-graft. Placement of supplementary bare metal stents to achieve complete expansion of the covered stent might be necessary, especially when using the self-expanding stent-grafts, which might be more prone compression by the aortic stent-graft.

\section{Limitations}

The main limitation of our study remains the fact that, in cases involving $>2$ side branches, the preferable option at Site 1 was to use a fenestrated devices in contrast to Site 2 , which used multiple chimneys. In this context, the results do not allow drawing any conclusion as to which repair technique is more appropriate to treat pararenal aortic pathologies. Additional relevant drawbacks of our study are the limited number of patients and the absence of long-term results.

\section{Conclusion}

Our series shows that the chimney technique applied to the one or both renal arteries is a safe and feasible method, with excellent results in terms of sac shrinkage and patency of the chimney and periscope grafts up to 24 months postoperatively. However, further clinical and radiological surveillance in a greater number of patients with longer follow-up will be needed to confirm these promising results.

\section{REFERENCES}

1. Donas KP, Torsello G, Bisdas T. New EVAR devices: pros and cons. J Cardiovasc Surg (Torino). 2012;53:559-569.

2. Donas KP, Pecoraro F, Torsello G, et al. Use of covered chimney stents for pararenal aortic pathologies is safe and feasible with excellent patency and low incidence of endoleaks. $J$ Vasc Surg. 2012;55:659-665.

3. Gehringhoff B, Torsello G, Pitoulias GA, et al. Use of chimney grafts in aortic arch pathologies involving the supra-aortic branches. J Endovasc Ther. 2011;18:650-655.

4. Donas KP, Eisenack M, Panuccio G, et al. The role of open and endovascular treatment with fenestrated and chimney endografts for patients with juxtarenal aortic aneurysms. $J$ Vasc Surg. 2012;56:285-290.

5. Mayer D, Rancic Z, Wilhelm M, et al. Improved hybrid technique for vascular access and closure. J Endovasc Ther. 2008;15:322-325.

6. Resch TA, Sonesson B, Dias N, et al. Chimney grafts: is there a need and will they work? Perspect Vasc Surg Endovasc Ther. 2011;23: 149-153.

7. Pecoraro F, Pfammatter T, Mayer D, et al. Multiple periscope and chimney grafts to treat ruptured thoracoabdominal and pararenal aortic aneurysms. J Endovasc Ther. 2011;18:642-649.

8. Amiot S, Haulon S, Becquemin JP, et al. Fenestrated endovascular grafting: the French multicentre experience. Eur $J$ Vasc Endovasc Surg. 2010;39:537-544.

9. Donas KP, Bisdas T, Torsello G, et al. Technical considerations and performance of bridging stent-grafts for iliac side branched devices based on a pooled analysis of single-center experiences. J Endovasc Ther. 2012;19:667-671. 